\title{
GPX7 promotes the growth of human papillary thyroid carcinoma via enhancement of cell proliferation and inhibition of cell apoptosis
}

\author{
Li-Dan Liu ${ }^{1,2}$, Yi-Ni Zhang ${ }^{2}$, Li-Fen Wang ${ }^{2}$ \\ ${ }^{1}$ Press of Journal, Dalian Medical University, Dalian 116044, China; ${ }^{2}$ Department of Pathology, The Second Affiliated Hospital of Dalian Medical \\ University, Dalian 116023, China \\ Contributions: (I) Conception and design: LF Wang (II) Administrative support: LF Wang; (III) Provision of study materials or patients: LF Wang; \\ (IV) Collection and assembly of data: LD Liu; (V) Data analysis and interpretation: YN Zhang; (VI) Manuscript writing: All authors; (VII) Final \\ approval of manuscript: All authors. \\ Correspondence to: Li-Fen Wang. Department of Pathology, The Second Affiliated Hospital of Dalian Medical University, No. 467 Zhongshan Road, \\ Shahekou District, Dalian 116023, China. Email: lifen_w@163.com.
}

\begin{abstract}
Background: Abnormal expression of glutathione peroxidase 7 (GPX7) has been linked to the occurrence and development of a variety of tumors. However, the role of GPX7 in the progression of papillary thyroid carcinoma (PTC) has not been elucidated.This study investigated the role of GPX7 in the progression of PTC.

Methods: The methods employed included immunohistochemistry, Western blotting, quantitative reverse transcription-polymerase chain reaction (qRT-PCR), MTT assay, Celigo cell counting, flow cytometric analysis, caspase activity assay, cell clone formation assay, and GPX7 knockdown.

Results: The data showed that GPX7 protein was localized in the cytoplasm of thyroid cells. The level of GPX7 expression was higher in PTC tissues than in the nodular goiter. The positive rate for GPX7 was also higher in the PTC group than in the nodular goiter group (100.0\% vs. 35.7\%). The maximum tumor diameter in the group highly expressing GXP7 was significantly greater than that in the group with low expression of GXP7 (1.56 \pm 0.56 vs. $0.56 \pm 0.13 \mathrm{~cm}, \mathrm{P}<0.001)$. The GPX7 mRNA level was higher in $\mathrm{K} 1$ cells. Knockdown of GPX7 decreased the number of cells, cell clone formation ability, and cell proliferation rate and increased the activity of caspase $3 / 7$ and cell apoptosis in PTC K1 cells.
\end{abstract}

Conclusions: These results indicate that high expression of GPX7 increases the proliferation and reduces the apoptosis of PTC cells, and thus, promotes the growth and progression of human PTC.

Keywords: Glutathione peroxidase 7 (GPX7); papillary thyroid carcinoma (PTC); cell proliferation; cell apoptosis

Submitted May 30, 2019. Accepted for publication Sep 26, 2019.

doi: $10.21037 /$ tcr.2019.10.14

View this article at: http://dx.doi.org/10.21037/tcr.2019.10.14

\section{Introduction}

Thyroid carcinoma is a common malignancy in the endocrine system that develops from the epithelial cells of the thyroid. The incidence of thyroid cancer is steadily rising worldwide. Thyroid cancers are mainly classified into papillary thyroid carcinoma (PTC), follicular thyroid carcinoma (FTC), medullary thyroid carcinoma, and undifferentiated thyroid carcinoma. PTC is among the differentiated thyroid carcinomas (DTCs) (1) and contributes most to the increased incidence of thyroid cancer. Although PTC generally is an indolent malignancy with favorable outcomes, PTC commonly invades the lymph nodes, with a frequency ranging from $30-80 \%$ of patients (2), and approximately $10 \%$ of PTC cases advance to dedifferentiated PTC, which is associated with a poor prognosis and high rate of early death (3). Therefore, a better understanding of the molecular mechanisms of PTC is still needed for the development of therapeutic 
interventions for PTC.

A number of genetic abnormalities as well as a few major signaling pathways have been found to be involved in tumorigenesis of PTC (4). It has also been proposed that reactivation of some pathways, such as epithelial to mesenchymal transition, change in microenvironment, and oncogenes of the thyroid, BRAF, RET/PTC, and Ras, may be involved in the tumorigenesis and migration of PTC (5-7). However, the molecular mechanisms of PTC remain unclear and need to be further investigated.

The oxidative stress response reflects an imbalance between the oxidation system and the anti-oxidation system caused by an excessive systemic manifestation of reactive oxygen species (ROS) following harmful stimuli over the body's ability to detoxify the reactive intermediates, thus leading to damage of tissue. ROS are natural products of multiple intrinsic processes. Some scholars have suggested that oxidative stress is related to the origin and progression of tumors. Oxidative stress is generated when an imbalance occurs between systemic production of ROS and the biological detoxification processes. ROS include $\mathrm{O}_{2}^{-}, \mathrm{H}_{2} \mathrm{O}_{2}, \mathrm{HO}_{2}^{-},-\mathrm{OH}$ and other active oxygen molecules (8). Persistent exposure to oxidative species brings about systemic disorders, such as autoimmunity, carcinogenesis, diabetes mellitus, obesity, neurodegeneration, and aging (9-13).

$\mathrm{H}_{2} \mathrm{O}_{2}$ is an essential substrate of thyroid peroxidase and a critical enzyme responsible for the synthesis of thyroid hormone, and it plays an important function in the synthesis of thyroid hormone and the physiological function of the thyroid $(14,15)$. Under abnormal conditions, $\mathrm{H}_{2} \mathrm{O}_{2}$ can accumulate in the thyroid gland, and the thyroid is thus persistently exposed to a high concentration of $\mathrm{H}_{2} \mathrm{O}_{2}$ and the resultant ROS environment. With the oxidative stress response, oxidative DNA damage or gene hypomethylation can happen within the thyroid cells (16). Therefore, abnormalities in the redox reaction may be related to the occurrence and development of PTC.

Living organisms have evolved to possess multiple mechanisms to resist oxidative stress. Catalase, peroxiredoxin, and glutathione peroxidase (GPX) are three important enzyme families involved in ROS removal (17). The GPXs are the most important oxidoreductases in mammalian cells. The GPX family consists of eight GPXs, named GPX1-GPX8, among which GPX1-GPX4 and GPX6 are selenocysteine-containing, and GPX5, GPX7, and GPX8 were non-selenocysteine-containing $(18,19)$. In general, selenocysteine-containing GPXs can reduce $\mathrm{H}_{2} \mathrm{O}_{2}$ via catalytic reduction of glutathione $(\mathrm{GSH})$ to reduce the oxidative stress response (17). The mechanisms of nonselenocysteine-containing GPXs of peroxidase activity are controversial, because of the lack of a GSH-binding domain. GPX7 is mainly involved in maintaining the redox homeostasis of the body. Recent studies reported that the abnormal expression of GPX7 is related to the occurrence and development of a variety of tumors, including esophageal adenocarcinoma, breast cancer, hepatocellular carcinoma (HCC), and others (20-22), but the results in different tumors were not consistent. For example, Peng et al. (20) found that GPX7 expression is absent in esophageal adenocarcinoma cells, suggesting that GPX7 may play a role in inhibiting the development of esophageal adenocarcinoma, and it may inhibit tumorigenesis through $\mathrm{NF}-\kappa \mathrm{B}$ signaling pathway. It was also reported that the function of GPX7 to inhibit tumorigenesis may be through the protective effect of oxidative stress (17). The Italian scholar Guerriero used immunohistochemistry and quantitative reverse transcription-polymerase chain reaction (qRT-PCR) to study the expression of GPX7 in HCC tissues. It was found that GPX7 was highly expressed in HCC tissues and the expression level in grade III HCC tissues was significantly higher than that in grade I-II. It suggested that the increase of GPX7 may play a role in the occurrence and development of HCC (22). However, the role of GPX7 in PTC has not been studied. Therefore, the present study was designed to uncover the role of GPX7 in PTC.

\section{Methods}

\section{Ethical approval}

This study was approved by the ethics committee of the Second Affiliated Hospital of Dalian Medical University. All procedures performed in studies involving human participants were in accordance with the ethical standards of the institutional and/or national research committee and with the 1964 Helsinki declaration and its later amendments or comparable ethical standards.

\section{Patients and PTC cell lines}

Forty-four patients (30 with PTC and 14 with nodular goiter) treated in the Second Hospital of Dalian Medical University from January 2015 to September 2016 were selected for enrollment in the study. After the pathologic 
Table 1 Antibodies information of immunohistochemistry

\begin{tabular}{lll}
\hline Antibody name & Antibody source & Cat. no. \\
\hline GPX7 polyclonal antibody & Wuhan San Ying Biotechnology Co., Ltd. & $13501-1-A P$ \\
Secondary antibody and DAB & Beijing Zhongshan Gold Bridge Biotechnology Co., Ltd. & 15102901 \\
\hline
\end{tabular}

GPX7, glutathione peroxidase 7 .

Table 2 Reagent and apparatus information of RT-PCR

\begin{tabular}{lll}
\hline Reagent name & Reagent source & Cat. no. \\
\hline TRlzol & Shanghai Pufei Biotech Co., Ltd. & $3101-100$ \\
M-MLV & Promega & M1705 \\
dNTPs & Promega & U1240 \\
oligo dT & Sangon Biotech (Shanghai) Co., Ltd. & B0205 \\
Rnase inhibitor & Promega & N2115 \\
SYBR master mixture & TAKARA & DRR041B \\
Real time PCR apparatus & Roche & LightCycler480 \\
\hline
\end{tabular}

RT-PCR, reverse transcription-polymerase chain reaction.

diagnosis was clearly made by two senior pathologists who reviewed the pathological sections, patients with other malignancies and incomplete clinical data were excluded. The clinicopathological data of the included patients were extracted from the records and used for this study.

The human PTC cell lines K1 was purchased from the European Collection of Cell Cultures.

\section{Immunobistochemistry}

Immunohistochemistry was performed using the MaxVision method, and GPX7 expression was detected with GPX7 polyclonal antibody (13501-1-AP, Wuhan San Ying Biotechnology Co., Ltd., Wuhan, China), secondary antibody and DAB (Beijing Zhongshan Gold Bridge Biotechnology Co., Ltd., Beijing, China) (Table 1). The positive signal for GPX7 expression in the cytoplasm was quantified by randomly observing five high power fields for each case and grading according to the percentage of stained cells and staining intensity. The expression level of GPX7 for a patient was scored according to the percentage of positive cells as follows: 0 for $<10 \%, 1$ for $11-25 \%, 2$ for $26-50 \%, 3$ for $51-$ $75 \%$, and 4 for $>75 \%$. Staining intensity was scored as: 0 for no staining, 1 for light yellow, 2 for brownish yellow, and 3 for brown. The final scores were generated by multiplication of the positive cell percentage score with the staining intensity score, resulting in final scores of: 0 for negative (-), 1-4 for weak positive (+), 5-8 for moderate positive (++), and 9-12 for strong positive (+++).

\section{qRT-PCR}

Total RNA was isolated from the K1 cell lines using the TRIzol reagent (Shanghai Pufei Biotech Co., Ltd., Shanghai, China) following the manufacturer's instructions. Single-stranded cDNA was synthesized using $2 \mu \mathrm{g}$ of total RNA from each sample as a template through reverse transcription (Table 2). GPX7 mRNA was detected by PCR using $1 \mu \mathrm{L}$ of cDNA as the template and glyceraldehyde-3phosphate dehydrogenase (GAPDH) as an internal standard. The primers used were as follows: GPX7 forward primer, 5'-CGCACCTACAGTGTCTCATTC-3' and GPX7 reverse primer, 5'-CAGGTACTTGAAGGCAGGATG-3'; GAPDH forward primer, 5'-TGA CTT CAA CAG CGA CAC CCA-3' and GAPDH reverse primer, 5'-CAC CCT GTT GCT GTA GCC AAA-3'. The PCR profile was: $95^{\circ} \mathrm{C}$ for $15 \mathrm{sec} ; 45$ cycles of $95^{\circ} \mathrm{C}$ for $5 \mathrm{sec}$ and $60^{\circ} \mathrm{C}$ for 30 sec. The PCR amplicons of GPX7 and GAPDH were 81 and $121 \mathrm{bp}$, respectively. All samples were tested in triplicate. 
Table 3 Reagent information of recombinant lentiviral vector preparation

\begin{tabular}{lcc}
\hline Reagent name & Reagent source & Cat. no. \\
\hline Agel & NEB & R3552L \\
EcoRI & NEB & R3101L \\
CutSmart buffer & NEB & B7204S \\
Polymerase & Vazyme & P201-D3 \\
T4 DNA ligase & Fermentas & EL0016 \\
TIANgel midi purification kit & TIANGEN & DP209-03 \\
EndoFree maxi plasmid kit & TIANGEN & DP117 \\
\hline
\end{tabular}

Table 4 Antibodies information of Western blotting

\begin{tabular}{lcc}
\hline Antibody name & Antibody source & Cat. no. \\
\hline Mouse anti-FLAG & Sigma & F1804 \\
Mouse anti-GAPDH & Santa Cruz & sc-32233 \\
Goat anti-mouse lgG & Santa Cruz & sc-2005 \\
\hline
\end{tabular}

GAPDH, glyceraldehyde-3-phosphate dehydrogenase.

\section{Recombinant lentiviral vector preparation and cell infection}

The DNA sequence (GCACCTACAGTGTCTCATT) complementary to the full-length GPX7 sequence (GenBank no. NM_015696) of GPX7 was designed by GeneChem Co., Ltd. (Shanghai, China). The oligonucleotides were synthesized and ligated into the lentivirus-based pGCSILGFP (GeneChem Co., Ltd.) at AgeI/EcoRI sites (23).

For lentivirus infection, GPX7-siRNA-lentivirus or negative control (NC) lentivirus was added to $\mathrm{K} 1$ cells cultured in 6-well plates according to a multiplicity of infection (MOI). Infection efficiency was monitored through observing the cells under a fluorescence microscope (MicroPublisher 3.3RTV; Olympus, Tokyo, Japan) after $72 \mathrm{~h}$ of infection. GPX7 knockdown efficiency was determined by qRT-PCR using the cells harvested after an infection period of $120 \mathrm{~h}$ (Table 3).

\section{Western blotting}

The mixture of plasmid DNA and Lipofectamine 2000 was added to $293 \mathrm{~T}$ cells and cultured in incubator containing $5 \% \mathrm{CO}_{2}$ at $37^{\circ} \mathrm{C}$ for $6-8 \mathrm{~h}$. Then the cells were transferred into fresh complete culture media containing $10 \%$ serum. After transfection for 36-48 h, the cells were collected, and total protein was extracted. An equal amount of total protein for each treatment was separated on $12.5 \%$ sodium dodecyl sulfate-polyacrylamide gel electrophoresis to Laemmli's method (24) and transferred onto polyvinylidene difluoride membranes. Membranes were incubated with mouse anti-FLAG (Sigma, St. Louis, MO, USA) or antiGAPDH antibodies (Santa Cruz Biotechnology, Dallas, TX, USA), and horseradish peroxidase-conjugated goat anti-mouse IgG (Santa Cruz Biotechnology). Signals were developed using enhanced chemiluminescence reagent (ECL-Plus/Kit; Amersham, Piscataway, NJ, USA) (Table 4).

\section{Celigo cell counting}

For cell counting, $100 \mu \mathrm{L}$ of cell suspension was plated into 96-well plates at a density of 2,000 cells/well in triplicate. Cells were cultured in a $5 \% \mathrm{CO}_{2}$ incubator at $37^{\circ} \mathrm{C}$. From the second day after inoculation, the Celigo assay was carried out daily for 5 consecutive days. The cells with green fluorescence were counted at each scanning. The proliferation rate at each time point of the group was generated by calculating the ratio of the number of cells at each time point to the initial number of cells on the first day and used to plot the growth curve against the time points (25).

\section{Flow cytometry}

Cells were divided into two groups and cultured in 6-well plates to $70 \%$ confluence. The cells were re-suspended with trypsin and harvested into $5-\mathrm{mL}$ centrifuge tubes in triplicate (number of cells $\geq 5 \times 10^{5} /$ treatment) for each group. The suspension was then centrifuged at $1,000 \mathrm{~g}$ for $5 \mathrm{~min}$, the supernatant was discarded, and the cell pellets were washed with D-Hanks pre-cooled at $4{ }^{\circ} \mathrm{C}$ and then centrifuged at 1,000 $\mathrm{g}$ for $3 \mathrm{~min}$. After the cell pellets were re-suspended in $200 \mu \mathrm{L}$ binding buffer, $10 \mu \mathrm{L}$ Annexin $\mathrm{V}$-APC was mixed with the suspension to stain the cells at room temperature in darkness for 10-15 min. Another $400-800 \mu \mathrm{L}$ binding buffer was added before detection (26).

\section{Caspase 3/7 assay}

Cells were inoculated onto 96-well plates and cultured for 5 days at $37{ }^{\circ} \mathrm{C}$ in a $\mathrm{CO}_{2}$ incubator. After the cells were suspended and the cell suspension was adjusted to $1 \times 10^{4}$ cells $/ \mathrm{mL}$ at room temperature, both the target cells and the NC cells were added to new 96-well plates at $100 \mu \mathrm{L}$ per well. Then $100 \mu \mathrm{L}$ of caspase-Glo reaction 
Table 5 The clinical information of PTC and nodular goiter group

\begin{tabular}{|c|c|c|c|c|}
\hline Parameters & PTC $(n=30)$ & $\begin{array}{l}\text { Nodular goiter } \\
\qquad(n=14)\end{array}$ & $\mathrm{F} / \mathrm{t}$ & $P$ \\
\hline Gender, n (\%) & & & 2.065 & 0.307 \\
\hline Female & 28 (93.3) & $11(78.6)$ & & \\
\hline Male & $2(6.7)$ & $3(21.4)$ & & \\
\hline Age, years & $44.97 \pm 12.53$ & $59.64 \pm 9.48$ & 15.091 & $<0.001$ \\
\hline $\begin{array}{l}\text { T3 expression, } \\
\text { n (\%) }\end{array}$ & & & 0.978 & 0.613 \\
\hline Normal & 28 (93.3) & $14(100.0)$ & & \\
\hline Elevate & $1(3.3)$ & 0 & & \\
\hline Reduce & $1(3.3)$ & 0 & & \\
\hline $\begin{array}{l}\text { T4 expression, } \\
\mathrm{n}(\%)\end{array}$ & & & 1.502 & 0.472 \\
\hline Normal & $27(90.0)$ & $14(100.0)$ & & \\
\hline Elevate & $1(3.3)$ & 0 & & \\
\hline Reduce & $2(6.7)$ & 0 & & \\
\hline $\begin{array}{l}\text { TSH expression, } \\
\mathrm{n}(\%)\end{array}$ & & & 3.700 & 0.157 \\
\hline Normal & $24(80.0)$ & $11(78.6)$ & & \\
\hline Elevate & 4 (13.3) & 0 & & \\
\hline Reduce & $2(6.7)$ & $3(21.4)$ & & \\
\hline
\end{tabular}

PTC, papillary thyroid carcinoma; TSH, thyroid-stimulating hormone.

solution was added to each well. The mixture was shaken for $30 \mathrm{~min}$ at $400 \mathrm{~g}$ in a shaker, and then incubated at $22{ }^{\circ} \mathrm{C}$ for $1.5 \mathrm{~h}$. The signal was measured by microplate reader (M2009PR, Tecan Infinite, Switzerland) (27).

\section{MTT assay}

Cells in the exponential growth phase were digested with trypsin and re-suspended in complete media. The cells were inoculated onto 96-well plates at 2,000 cells/well in triplicate for each group and cultured for 5 days. The cells were transferred to five 96-well plates and incubated in a cell incubator. On the second day after plating, $20 \mu \mathrm{L}$ of $5 \mathrm{mg} / \mathrm{mL}$ MTT solution was delivered to each well $4 \mathrm{~h}$ before the culture was terminated. The medium was completely blotted, and formazan particles were dissolved by addition of $100 \mu \mathrm{L}$ DMSO. The mixture was stirred on an oscillator for 2-5 min, and the optical density
(OD) value was detected using a microplate reader at excitation/emission wavelengths of 490/570 nm (28).

\section{Clone formation assay}

Cells were inoculated onto 6-well plates at 800 cells/well in triplicate for each group. The cells were cultured in an incubator for 11 days with media changed and cells observed every 3 days. After the cell clones were photographed under a fluorescence microscope before termination of the experiment, the cells were washed once with phosphatebuffered saline (PBS). After the cells were fixed in $1 \mathrm{~mL}$ of $4 \%$ paraformaldehyde for $60 \mathrm{~min}$, the cells were washed once with PBS and stained with $500 \mu \mathrm{L}$ clean inclusion-free GIEMSA (AR-0752, Shanghai Dingguo Biotechnology Co., Ltd., Shanghai, China) for $20 \mathrm{~min}$. The cells were washed with $\mathrm{ddH}_{2} \mathrm{O}$ several times, dried in air, and photographed using digital camera, and the cell clones were counted (29).

\section{Statistical analysis}

Statistical analysis was performed using Statistical software (IBM SPSS Statistics 20). mRNA expression values of the genes were analyzed via the $2^{-\Delta \mathrm{Ct}}$ method, and data are expressed as mean \pm standard deviation. Student's $t$-test was used to determine the significance of the differences in all tested parameters between the groups. $\mathrm{P}<0.05$ was taken as statistically significant.

\section{Results}

\section{The clinical information of PTC and nodular goiter group}

From the comparison of the clinical information between PTC and nodular goiter group, it could be demonstrated that there was no statistical difference between the two groups except age (Table 5).

\section{GPX7 Expression in PTC tissue and cell lines}

The expression of GPX7 was detected in the PTC tissues and nodular goiters by immunohistochemistry. Our data demonstrated that GPX7 expression was localized in the cytoplasm of thyroid cells. The level of GPX7 expression was higher in PTC tissues than in the nodular goiter (Figure 1A). The positivity rate of GPX7 was $100.0 \%$ (mainly moderately to strongly positive) in the PTC group, while it was only $35.7 \%$ in the nodular goiter group (all weakly positive; Figure 1A, Table 6). According to the 

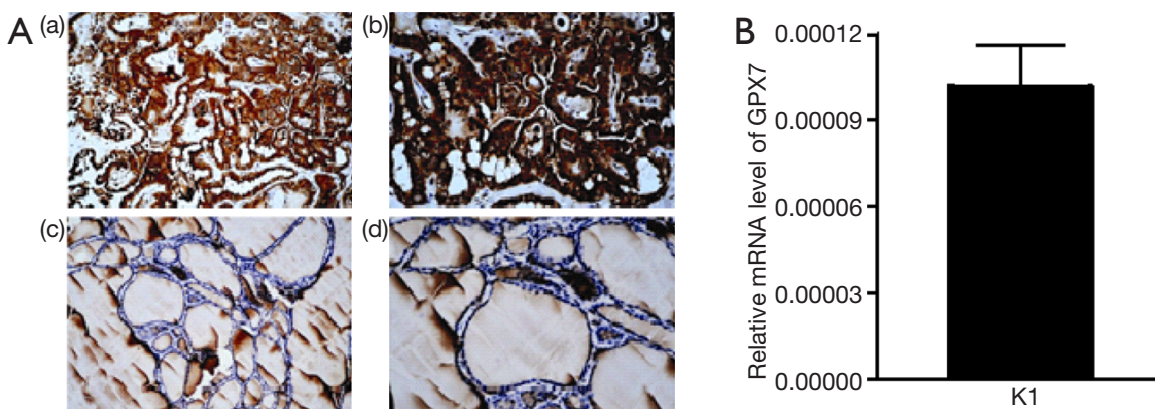

Figure 1 Expression of GPX7 in PTC tissue and cell lines. (A) Expression of GPX7 as detected by immunohistochemical staining in PTC and nodular goiter samples; (a) PTC group (×200); (b) PTC group (×400); (c) nodular goiter group (×200); (d) nodular goiter group ( $\times 400)$; (B) expression of GPX7 mRNA in different PTC cells. GPX7, glutathione peroxidase 7; PTC, papillary thyroid carcinoma.

Table 6 Expression of GPX7 in the thyroid tissues

\begin{tabular}{|c|c|c|c|c|c|c|}
\hline \multirow{2}{*}{ Group } & \multirow{2}{*}{ Scores $(\bar{X} \pm \mathrm{S})$} & \multicolumn{4}{|c|}{ Classification of GPX7 expression, n (\%) } & \multirow{2}{*}{ Positive rate $(\%)$} \\
\hline & & - & + & ++ & +++ & \\
\hline PTC $(n=30)$ & $8.13 \pm 2.46$ & 0 & $5(16.7)$ & $19(63.3)$ & $6(20.0)$ & 100.0 \\
\hline Nodular goiter $(n=14)$ & $1.07 \pm 1.59$ & $9(64.3)$ & $5(35.7)$ & 0 & 0 & 35.7 \\
\hline
\end{tabular}

GPX7, glutathione peroxidase 7; PTC, papillary thyroid carcinoma.

expression level of GPX7, PTC cases were divided into a high GPX7 expression $(\geq++)$ group $(\mathrm{n}=25)$ and a low GPX7 expression (+) group $(\mathrm{n}=5)$. The maximum tumor diameter $(1.56 \pm 0.56 \mathrm{~cm})$ of the high GXP7 expression group was significantly greater than that of the low GXP7 expression group $(0.56 \pm 0.13 \mathrm{~cm}, \mathrm{P}<0.001)$. The other clinical pathological parameters showed no statistically significant differences between the groups (Table 7). The high expression of GPX7 in K1 cell lines was detected by real-time PCR (the relative mRNA level of GPX7 was $1.02 \times 10^{-4} \pm 1.45 \times 10^{-5}$; Figure $\left.1 B\right)$.

\section{Expression of GPX7 in K1 cells transfected with lentivirus generating GPX7 RNAi}

To explore the potential roles of GPX7 in K1 cells, we knocked down GPX7 in K1 cells via lentivirus-mediated gene transfer. As indicated by the fluorescent signals, K1 cells were infected by the recombinant lentivirus containing shRNA targeting GPX7 (shGPX7) or control shRNA (shCtrl) with high efficiency (Figure 2A). The mRNA level of GPX7 was significantly lower in shGPX7-infected K1 cells than in shCtrl-transfected K1 cells $(\mathrm{P}<0.001$; Figure $2 B)$. The level of GPX7 protein expression also was dramatically reduced in shGPX7-infected cells compared with that in shCtrltransfected cells (Figure 2C). These results indicated that the recombinant lentivirus carrying shGPX7 could effectively down-regulate the expression of GPX7 in K1 cells.

\section{Effects of GPX7 knockdown on cell proliferation and colony formation ability of $\mathrm{K} 1$ cells}

To examine the influence of GPX7 on cell proliferation, we measured cell growth by Celigo cell counting, MTT assay, and cell clone formation in shCtrl- and shGPX7infected K1 cells. As shown in Figure 3A, the proliferation of shGPX7-infected K1 cells was significantly inhibited compared to that of shCtrl-infected $\mathrm{K} 1$ cells, as indicated by both cell counts and the proliferation rate $(\mathrm{P}<0.05)$. The MTT assay results indicated that the cell proliferation rate [presented as the ratio of $\mathrm{OD}_{490}$ value on day 5 to day 1] was significantly lower for the shGPX7-infected K1 cells than the shCtrl-infected K1 cells $(3.081 \pm 0.160$ vs. $4.897 \pm 0.091$, $\mathrm{P}<0.05$; Figure $3 B, C, D, E)$. The number of clones was significantly lower in the shGPX7-infected $\mathrm{K} 1$ cell samples than in the shCtrl-infected K1 cell samples $(283 \pm 6 v$ s. $710 \pm 10, \mathrm{P}<0.05$; Figure $3 F, G)$. These results demonstrated that down-regulation of GPX7 significantly inhibited the proliferation of $\mathrm{K} 1$ cells. 
Table 7 Relationships between GPX7 expression and clinical pathological parameters of PTC

\begin{tabular}{|c|c|c|c|c|}
\hline Parameters & High GPX7 expression $(n=25)$ & Low GPX7 expression $(n=5)$ & $\mathrm{t}$ & $\mathrm{P}$ \\
\hline Gender, n [\%] & & & 1.000 & 0.690 \\
\hline Female & $23[92]$ & 5 [100] & & \\
\hline Male & $2[8]$ & 0 & & \\
\hline Unilateral & $9[36]$ & $4[80]$ & & \\
\hline Bilateral & 16 [64] & $1[20]$ & & \\
\hline Lymph node metastasis, $\mathrm{n}$ [\%] & & & 1.000 & 0.696 \\
\hline Yes & 15 [60] & $3[60]$ & & \\
\hline Yes & $17[68]$ & $2[40]$ & & \\
\hline No & $8[32]$ & $3[60]$ & & \\
\hline
\end{tabular}

GPX7, glutathione peroxidase 7; PTC, papillary thyroid carcinoma.

A
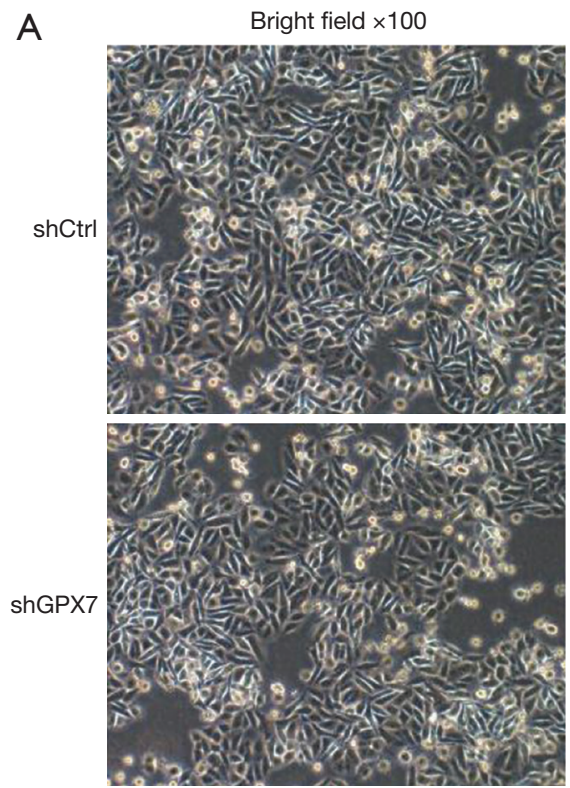

Fluorescent field $\times 100$
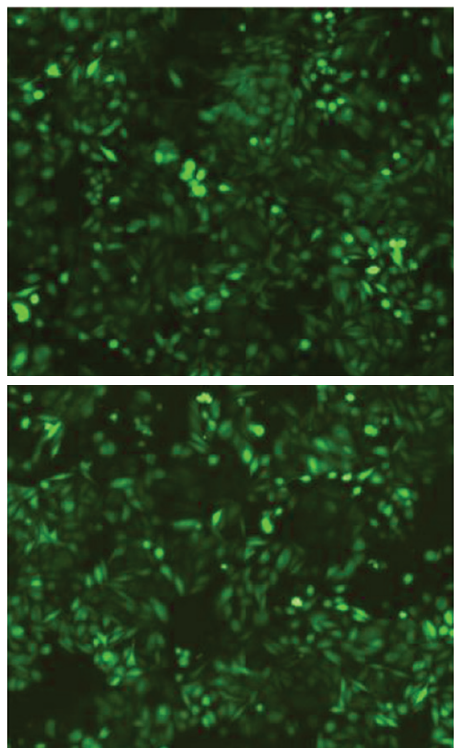

B

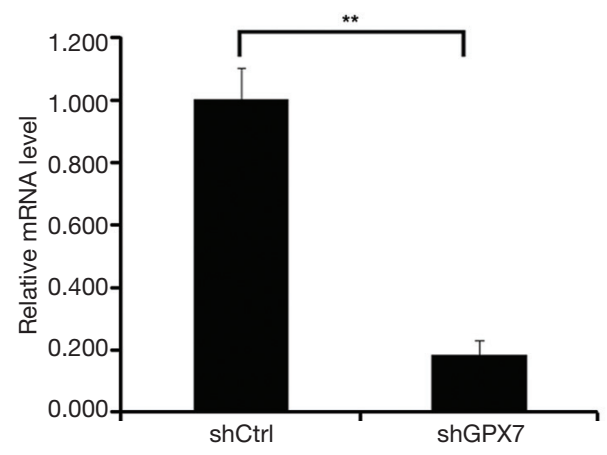

C

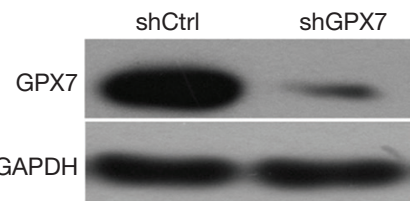

Figure 2 Expression of GPX7 in K1 cells after transfection with shGPX7. (A) Images of shCtrl- and shGPX7-infected K1 cells captured under fluorescence microscopy; left, bright field; right, fluorescence; (B) mRNA levels of GPX7 in shCtrl- and shGPX7-infected K1 cells; (C) protein levels of GPX7 in shCtrl- and shGPX7-infected K1 cells. ${ }^{*}, \mathrm{P}<0.001$. GPX7, glutathione peroxidase 7; shGPX7, shRNA targeting GPX7; shCtrl, control shRNA; GAPDH, glyceraldehyde-3-phosphate dehydrogenase. 


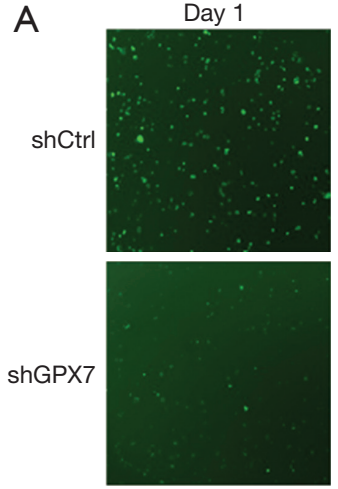

B

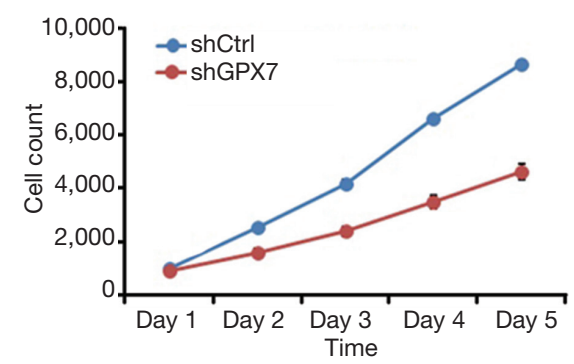

D
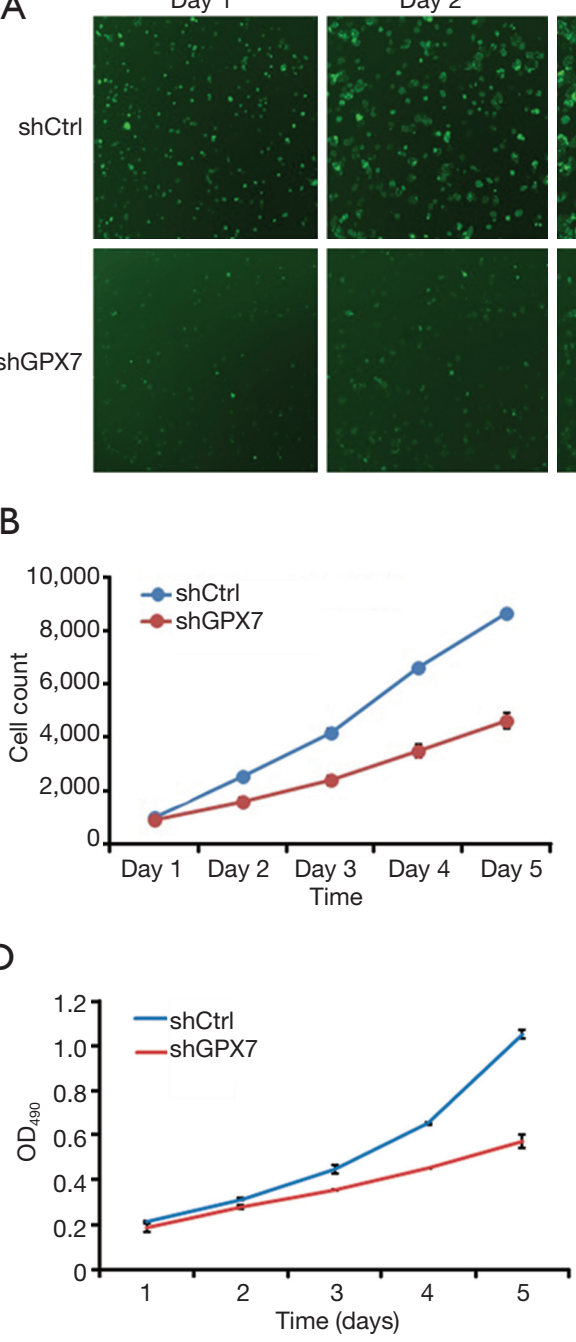

Day 3
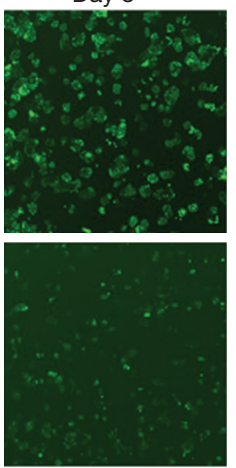

C

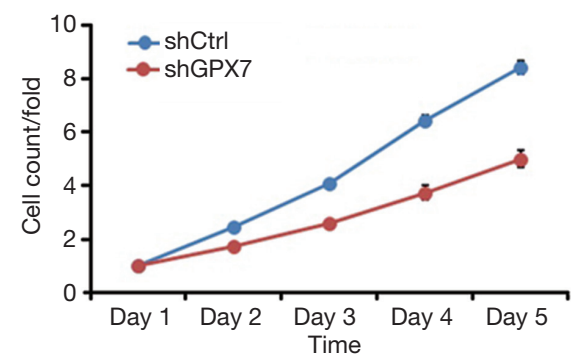

E

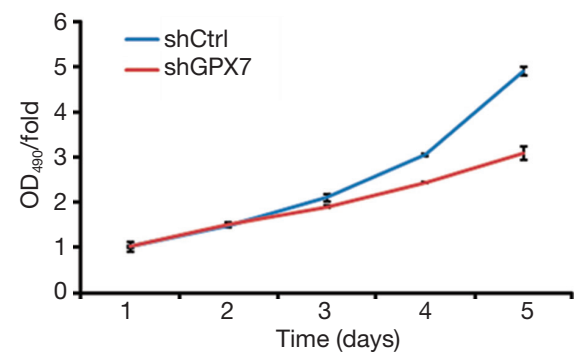

G

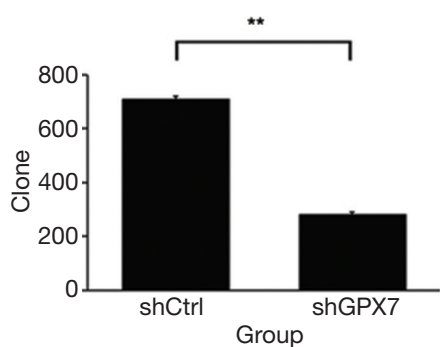

Figure 3 Cell proliferation and colony formation ability of K1 cells after GPX7 knockdown. (A) Images of proliferating cells in Celigo assay (×100); (B,C) cell counts and growth curves for shCtrl- and shGPX7-infected K1 cells; (D,E) growth curves for shCtrl- and shGPX7infected $\mathrm{K} 1$ cells evaluated at $\mathrm{OD}_{490}$; $\mathrm{F}$ ) representative images of cell colonies; $(\mathrm{G})$ comparison of the number of clones formed by shCtrland shGPX7-infected K1 cells. **, P<0.001. GPX7, glutathione peroxidase 7; shGPX7, shRNA targeting GPX7; shCtrl, control shRNA; $\mathrm{OD}_{490}$, optical density at $490 \mathrm{~nm}$. 


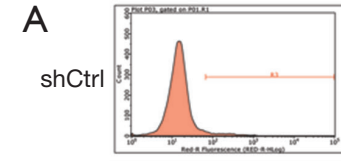

ANNEXIN-V

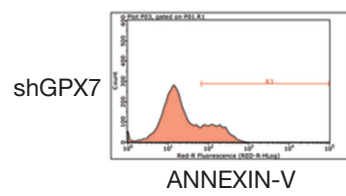

ANNEXIN-V

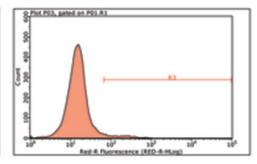

ANNEXIN-V

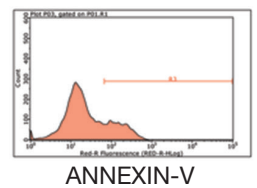

ANNEXIN-V

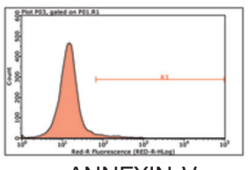

ANNEXIN-V

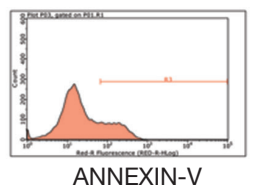

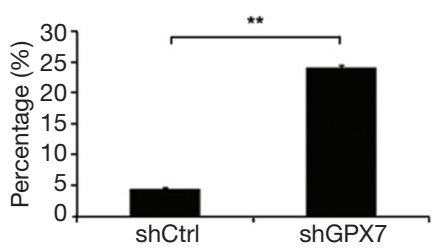

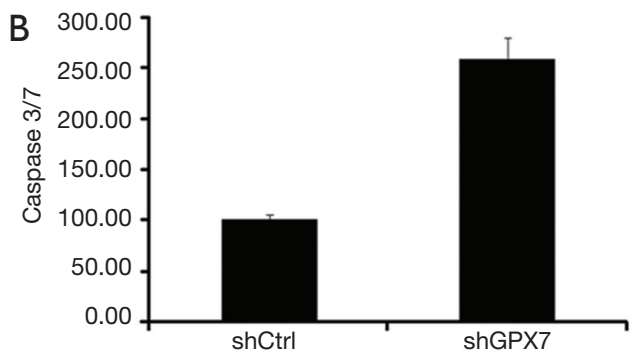

Figure 4 Knockdown of GPX7 increased cell apoptosis and altered the activity of apoptotic proteins in K1 cells. (A) Apoptosis among shCtrl- and shGPX7-infected K1 cells; (B) caspase 3/7 activity in shCtrl- and shGPX7-infected K1 cells. **, P<0.001. GPX7, glutathione peroxidase 7; shGPX7, shRNA targeting GPX7; shCtrl, control shRNA.

\section{Effects of GPX7 knockdown on cell apoptosis and the expression of apoptotic proteins in $\mathrm{K} 1$ cells}

To further detect the influence of GPX7 on cell apoptosis, we used flow cytometry to measure the percentage of dead cells among shCtrl- and shGPX7-infected K1 cells. The cell apoptosis percentage was significantly higher in shGPX7-infected K1 cells than in shCtrl-infected $\mathrm{K} 1$ cells $(24.08 \% \pm 0.28 \%$ vs. $4.33 \% \pm 0.15 \%, \mathrm{P}<0.001$; Figure $4 A$ ). Caspase $3 / 7$ activity was significantly higher in shGPX7-infected $\mathrm{K} 1$ cells than in shCtrl-infected $\mathrm{K} 1$ cells $(257.82 \pm 21.28$ vs. $100.00 \pm 5.38, \mathrm{P}<0.05$; Figure $4 B)$. These results demonstrated that down-regulation of GPX7 increased $\mathrm{K} 1$ cell apoptosis.

\section{Discussion}

In this study, GPX7 was found to be expressed at higher levels in PTC tissues and PTC cell lines than in other thyroid tissues and related to the size of PTC tumors. GPX7 was successfully knocked down in K1 cells, and knockdown of GPX7 inhibited cell proliferation and clone formation as well as increased apoptosis and caspase 3/7 activity in K1 cells. These results demonstrated that GPX7 promotes the growth of PTC.

In the present study, it was found that GPX7 expression was mainly localized in the cytoplasm of thyroid epithelial cells. The positive rate of GPX7 expression was 100.0\% (mainly moderately to strongly positive) in PTC samples, which was significantly higher than that in nodular goiter samples. GPX7 was also found to be highly expressed in the human PTC cell lines TPC-1 and K1. These results are in agreement with the observations that the abnormal expression of GPX7 is involved in the occurrence and development of a variety of tumors (including esophageal adenocarcinoma, breast cancer, HCC, etc.) (20-22). GPX is the most important oxidoreductase in the body (30) and critical for the maintenance of the body's redox homeostasis. Abnormal expression of GPX7 in PTC tissues and PTC cell lines supports the hypothesis that oxidative stress is somehow responsible for the occurrence and development of tumors $(31,32)$ and oxidative stress may be a risk factor for the development of thyroid cancer. The coincidence of a higher level of GPX7 expression with a larger maximum diameter of PTC tumors suggests that GPX7 may enhance the growth of PTC tumors.

Cells of the PTC K1 cell line were transfected with lentivirus carrying GPX7 RNAi, and both qPCR and Western blotting analyses showed that GPX7 expression was dramatically knocked down in the transfected K1 cells. Knockdown of GPX7 reduced the cell proliferation rate 
and cell clone formation ability and increased the activity of caspase 3/7 and apoptosis in $\mathrm{K} 1$ cells, suggesting that high expression of GPX7 in PTC could significantly reduce apoptosis and increase the proliferation of cells, which is in accordance with the role of GPX7 in HCC tissue reported by Guerriero (22). These results indicate that high expression of GPX7 in PTC can promote cell proliferation and decrease cell apoptosis, which leads to faster tumor growth and corresponds with our observation of a larger maximum diameter in PTC tumors with high GPX7 expression.

However, the mechanism of action of GPX7 on PTC proliferation and apoptosis is still unclear. Studies had shown that the activation of NF- $\mathrm{KB}$ was the sensor of oxidative stress (33). Peng et al. reported that the role of GPX7 in esophageal adenocarcinoma is through the NF- $\kappa \mathrm{B}$ pathway involved in tumorigenesis, suggesting that GPX7 may promote NF- $\mathrm{\kappa B}$ expression by phosphorylating NF$\kappa \mathrm{B}$ upstream kinase IKK- $\alpha / \beta$ or IкB- $\alpha$ (20). And, Pyo et $a l$. found that there was high expression of NF- $\mathrm{KB}$ p 65 in PTC, and its high expression was related to the occurrence and development of PTC (34). Then whether the effect of GPX7 on PTC proliferation and apoptosis is also achieved through the NF- $\kappa \mathrm{B}$ signaling pathway, further research is needed.

\section{Conclusions}

GPX7 was highly expressed in PTC tissue and cell lines, and such high expression of GPX7 could promote proliferation and reduce apoptosis among PTC cells, thereby promoting the growth of PTC.

\section{Acknowledgments}

Funding: None.

\section{Footnote}

Conflicts of Interest: All authors have completed the ICMJE uniform disclosure form (available at http://dx.doi. org/10.21037/tcr.2019.10.14). The authors have no conflicts of interest to declare.

Ethical Statement: The authors are accountable for all aspects of the work in ensuring that questions related to the accuracy or integrity of any part of the work are appropriately investigated and resolved. This study was approved by the ethics committee of the Second Affiliated Hospital of Dalian Medical University (No. 2019-077). All procedures performed in studies involving human participants were in accordance with the ethical standards of the institutional and/or national research committee and with the 1964 Helsinki declaration and its later amendments or comparable ethical standards. Individual informed consent was waived.

Open Access Statement: This is an Open Access article distributed in accordance with the Creative Commons Attribution-NonCommercial-NoDerivs 4.0 International License (CC BY-NC-ND 4.0), which permits the noncommercial replication and distribution of the article with the strict proviso that no changes or edits are made and the original work is properly cited (including links to both the formal publication through the relevant DOI and the license). See: https://creativecommons.org/licenses/by-nc-nd/4.0/.

\section{References}

1. Reiners C. Thyroid cancer in 2013: advances in our understanding of differentiated thyroid cancer. Nat Rev Endocrinol 2014;10:69-70.

2. Pereira JA, Jimeno J, Miquel J, et al. Nodal yield, morbidity, and recurrence after central neck dissection for papillary thyroid carcinoma. Surgery 2005;138:1095-100, discussion 1100-1.

3. Chen $\mathrm{H}$, Luo D, Zhang L, et al. Restoration of $\mathrm{p} 53$ using the novel MDM2-p53 antagonist APG115 suppresses dedifferentiated papillary thyroid cancer cells. Oncotarget 2017;8:43008-22.

4. Xie J, Fan Y, Zhang X. Molecular mechanisms in differentiated thyroid cancer. Front Biosci (Landmark Ed) 2016;21:119-29.

5. Kimura ET, Nikiforova $M N$, Zhu $Z$, et al. High prevalence of BRAF mutations in thyroid cancer: genetic evidence for constitutive activation of the RET/PTC-RAS-BRAF signaling pathway in papillary thyroid carcinoma. Cancer Res 2003;63:1454-7.

6. Xing M. Molecular pathogenesis and mechanisms of thyroid cancer. Nat Rev Cancer 2013;13:184-99.

7. Nikiforov YE. RET/PTC rearrangement in thyroid tumors. Endocr Pathol 2002;13:3-16.

8. Schieber $M$, Chandel NS. ROS function in redox signaling and oxidative stress. Curr Biol 2014;24:R453-62.

9. Li S, Zhu G, Yang Y, et al. Oxidative stress-induced chemokine production mediates $\mathrm{CD} 8(+) \mathrm{T}$ cell skin trafficking in vitiligo. J 
Investig Dermatol Symp Proc 2015;17:32-3.

10. Sosa V, Moliné T, Somoza R, et al. Oxidative stress and cancer: an overview. Ageing Res Rev 2013;12:376-90.

11. Matsuda M, Shimomura I. Increased oxidative stress in obesity: implications for metabolic syndrome, diabetes, hypertension, dyslipidemia, atherosclerosis, and cancer. Obes Res Clin Pract 2013;7:e330-41.

12. Uttara B, Singh AV, Zamboni P, et al. Oxidative stress and neurodegenerative diseases: a review of upstream and downstream antioxidant therapeutic options. Curr Neuropharmacol 2009;7:65-74.

13. Rascón B, Harrison JF. Lifespan and oxidative stress show a non-linear response to atmospheric oxygen in Drosophila. J Exp Biol 2010;213:3441-8.

14. Dumont JE, De Deken X, Miot F, et al. H2O2, signal, substrate, mutagen and chemorepellent from physiology to biochemistry and disease. Bull Mem Acad R Med Belg 2010;165:231-4; discussion 235.

15. Carvalho DP, Dupuy C. Thyroid hormone biosynthesis and release. Mol Cell Endocrinol 2017;458:6-15.

16. Versteyhe S, Driessens N, Ghaddhab C, et al. Comparative analysis of the thyrocytes and T cells: responses to $\mathrm{H} 2 \mathrm{O} 2$ and radiation reveals an $\mathrm{H} 2 \mathrm{O} 2$-induced antioxidant transcriptional program in thyrocytes. J Clin Endocrinol Metab 2013;98:E1645-54.

17. Chen YI, Wei PC, Hsu JL, et al. NPGPx (GPx7): a novel oxidative stress sensor/transmitter with multiple roles in redox homeostasis. Am J Transl Res 2016;8:1626-40.

18. Toppo S, Vanin S, Bosello V, et al. Evolutionary and structural insights into the multifaceted glutathione peroxidase (Gpx) superfamily. Antioxid Redox Signal 2008;10:1501-14.

19. Armstrong RN, Morgenstern R, Board PG. Glutathione transferases. In: McQueen CA. Comprehensive toxicology. 3rd ed. Oxford: Elsevier, 2018.

20. Peng DF, Hu TL, Soutto M, et al. Loss of glutathione peroxidase 7 promotes TNF- $\alpha$-induced $\mathrm{NF}-\kappa \mathrm{B}$ activation in Barrett's carcinogenesis. Carcinogenesis 2014;35:1620-8.

21. Utomo A, Jiang X, Furuta S, et al. Identification of a novel putative non-selenocysteine containing phospholipid hydroperoxide glutathione peroxidase (NPGPx) essential for alleviating oxidative stress generated from polyunsaturated fatty acids in breast cancer cells. J Biol Chem 2004;279:43522-9.

22. Guerriero E, Capone F, Accardo M, et al. GPX4 and GPX7 over-expression in human hepatocellular carcinoma tissues. Eur J Histochem 2015;59:2540.

23. Liu H, Liang S, Yang X, et al. RNAi-mediated RPL34 knockdown suppresses the growth of human gastric cancer cells. Oncol Rep 2015;34:2267-72.

24. Laemmli UK. Cleavage of structural proteins during the assembly of the head of bacteriophage T4. Nature 1970;227:680-5.

25. Nabzdyk CS, Chun M, Pradhan L, et al. High throughput RNAi assay optimization using adherent cell cytometry. J Transl Med 2011;9:48.

26. Pan Y, Shan W, Fang H, et al. Sensitive and visible detection of apoptotic cells on Annexin- $\mathrm{V}$ modified substrate using aminophenylboronic acid modified gold nanoparticles (APBA-GNPs) labeling. Biosens Bioelectron 2014;52:62-8.

27. Mooney LM, Al-Sakkaf KA, Brown BL, et al. Apoptotic mechanisms in T47D and MCF-7 human breast cancer cells. Br J Cancer 2002;87:909-17.

28. Stockert JC, Blázquez-Castro A, Cañete M, et al. MTT assay for cell viability: intracellular localization of the formazan product is in lipid droplets. Acta Histochem 2012;114:785-96.

29. Liu JW, Nagpal JK, Sun W, et al. ssDNA-binding protein 2 is frequently hypermethylated and suppresses cell growth in human prostate cancer. Clin Cancer Res 2008;14:3754-60.

30. Mills GC. Hemoglobin catabolism. I. Glutathione peroxidase, an erythrocyte enzyme which protects hemoglobin from oxidative breakdown. J Biol Chem 1957;229:189-97.

31. Ameziane-El-Hassani R, Schlumberger M, Dupuy C. NADPH oxidases: new actors in thyroid cancer? Nat Rev Endocrinol 2016;12:485-94.

32. Schmutzler C, Mentrup B, Schomburg L, et al. Selenoproteins of the thyroid gland: expression, localization and possible function of glutathione peroxidase 3. Biol Chem 2007;388:1053-9.

33. Li N, Karin M. Is NF-kappaB the sensor of oxidative stress? FASEB J 1999;13:1137-43.

34. Pyo JS, Kang G, Kim DH, et al. Activation of nuclear factor- $\kappa \mathrm{B}$ contributes to growth and aggressiveness of papillary thyroid carcinoma. Pathol Res Pract 2013;209:228-32.

Cite this article as: Liu LD, Zhang YN, Wang LF. GPX7 promotes the growth of human papillary thyroid carcinoma via enhancement of cell proliferation and inhibition of cell apoptosis. Transl Cancer Res 2019;8(7):2570-2580. doi: 10.21037/ tcr.2019.10.14 